\title{
The Top Ten Tips for Completing the USDA Annual Report
}

Robert A. Willems, DVM and

Joseph A. Nelson

From choosing the wrong pain categories, to sloppy arithmetic, there

are a number of potential pitfalls when completing and filing a USDA annual report. The authors offer clarification and guidance to make the process easier.
The authors are in the USDA/APHIS/AC Eastern Region Office, 920 Main Campus Dr., Ste. 200, Raleigh, NC 27606. Please address correspondence to Willems at Robert.A.Willems@aphis.usda.gov.
Every year each research facility registered with the United States Department of Agriculture (USDA) under the Animal Welfare Act (AWA) must submit an annual report to the USDA listing the number of animals used in studies by that institution during the previous year. Here are ten suggestions to help the research community properly fill out the report and avoid some of the more common mistakes. We hope that these tips will make it easier for researchers to fulfill their annual reporting requirements.

1. If you are using the paper form of the annual report, please use the Animal and Plant Health Inspection Service (APHIS) Form 7023 provided to you. Do not submit your own version of the form. Submit the original only. Copies are not necessary .

2. If you choose to use the electronic version of the annual report from the internet, you must request a new password each year from APHIS's Animal Care (AC) staff. Passwords from previous years will not work. Either AC Regional Office in Raleigh, NC (tel: 919-8557100) or Ft. Collins, CO (tel: 970-494-7478) can assist you with instructions on how to submit your Annual Report via the internet.

3. It is not necessary to include animals on the report that are not regulated under the AWA, such as laboratory rats and mice, birds, fish, amphibians, reptiles, farm animals used in agricultural research, or freeliving wild animals involved in research meeting the definition of a field study. If you wish to include these animals voluntarily, please do so at the end of the report, and label that section "Nonregulated Animals."

4. Consolidate the numbers to be reported from the various sites operated by your registered facility on a single submitted form. Do not send in a separate form for each site at which the facility used animals in the previous year. Instead, attach to the report a statement listing the location of all facilities or sites at which animals were used during the previous year.

5. Check your arithmetic. The totals listed in column F should equal the sum of those animals listed in columns C, D, and E. Do not include the numbers from column B in these totals.

6. Enter animals into the correct columns for their pain category. For example, you should enter in column D, not column E, animals for which pain relief was provided during the study. Enter in column $\mathrm{C}$ animals that experienced no pain or only slight or momentary pain, as from an injection.

7. All animals listed in column E require both a description of the procedure causing the pain and/or distress and an explanation of why relief from the pain and/or distress was not provided. A description of the procedure alone is not sufficient. Attach the description and explanation to the reporting form.

8. If your Institutional Animal Care and Use Committee (IACUC) has approved any exceptions to the AWA standards during the previous year, you must attach a summary of those exceptions to the annual report. For example, if the IACUC approved the temporary housing of an animal in a cage with smaller floor space than that required by the regulations for that animal so as to meet the scientific requirements of the study, then that is an exception to the standards and you must report it.

9. The annual report should be signed and dated by the Chief Executive Officer (CEO) or Institutional Official (IO) of the institution, as listed on the institution's USDA registration form. The submitted form must bear the original signature of the IO or CEO.

10 . Be sure your annual report is sub- 